\title{
POLYNOMIAL VECTOR FIELDS ON THE CLIFFORD TORUS
}

\author{
JAUME LLIBRE AND ADRIAN C. MURZA
}

\begin{abstract}
First we characterize all the polynomial vector fields in $\mathbb{R}^{4}$ which have the Clifford torus as an invariant surface. After we study the number of invariant meridians and parallels that such polynomial vector fields can have in function of the degree of these vector fields.
\end{abstract}

\section{Introduction AND STATEMENT OF the MAIN RESUltS}

The Clifford torus

$$
\mathbb{T}=\left\{\left(x_{1}, x_{2}, x_{3}, x_{4}\right) \in \mathbb{R}^{4}: x_{1}^{2}+x_{2}^{2}=\frac{1}{2}, x_{3}^{2}+x_{4}^{2}=\frac{1}{2}\right\}
$$

in geometric topology is the simplest and most symmetric Euclidean space embedding of the cartesian product of two circles. It lives in $\mathbb{R}^{4}$, as opposed to $\mathbb{R}^{3}$.

In MathSciNet at July 22 of 2017 it appears with the keyword "Clifford torus" 430 references. The more recent reference is [7]. In the reference [6] are studied the meridians of the surfaces of revolution and some information about the meridians of the Clifford torus can be found there. In the references [2, 12] are studied the parallels of the surfaces of revolutions and again contains some information on the parallels of the Clifford torus.

In this paper first we shall study the polynomial vector fields of arbitrary degree in $\mathbb{R}^{4}$ having the Clifford torus invariant by their flow, and after we shall compute the maximal number of parallels and meridians that a polynomial vector field of a given degree can exhibit on the Clifford torus.

The maximum number of invariant hyperplanes that a polynomial vector field in $\mathbb{R}^{n}$ can have in function of its degree was given in [8]. The

2010 Mathematics Subject Classification. Primary: 34C07, 34C05, 34C40.

Key words and phrases. invariant parallels, invariant meridians, polynomial vector field, Clifford torus. 
analogous result for the invariant straight lines of polynomial vector fields in $\mathbb{R}^{2}$ was provided before in [1]. The study of the maximum number of meridians and parallels for a torus in $\mathbb{R}^{3}$ were studied in $[9$, and for an algebraic torus in [10]. In surfaces of revolution in $\mathbb{R}^{3}$ the meridians and parallels invariant by polynomial vector fields have been studied in [5].

As usual we denote by $\mathbb{R}\left[x_{1}, x_{2}, x_{3}, x_{4}\right]$ the ring of the polynomials in the variables $x_{1}, x_{2}, x_{3}$ and $x_{4}$ with real coefficients. By definition a polynomial differential system in $\mathbb{R}^{4}$ is a system of the form

$$
\frac{d x_{i}}{d t}=P_{i}\left(x_{1}, x_{2}, x_{3}, x_{4}\right), \quad \text { for } i=1,2,3,4,
$$

where $P_{i}\left(x_{1}, x_{2}, x_{3}, x_{4}\right) \in \mathbb{R}\left[x_{1}, x_{2}, x_{3}, x_{4}\right]$. If $m_{i}$ is the degree of the polynomial $P_{i}$, then $m=\max \left\{m_{1}, m_{2}, m_{3}, m_{4}\right\}$ is the degree of the polynomial differential system (11).

We denote by

$$
\mathcal{X}=\sum_{i=1}^{4} P_{i}\left(x_{1}, x_{2}, x_{3}, x_{4}\right) \frac{\partial}{\partial x_{i}},
$$

the polynomial vector field associated to the differential polynomial system (11) of degree $m$.

An invariant algebraic hypersurface for the polynomial differential system (1) or for the polynomial vector field (2) is an algebraic surface $f=f\left(x_{1}, x_{2}, x_{3}, x_{4}\right)=0$ with $f \in \mathbb{R}\left[x_{1}, x_{2}, x_{3}, x_{4}\right]$, such that for some polynomial $K \in \mathbb{R}\left[x_{1}, x_{2}, x_{3}, x_{4}\right]$ we have

$$
\mathcal{X} f=K f .
$$

Therefore if a solution curve of system (1) has a point on the algebraic hypersurface $f=0$, then the whole solution curve is contained in $f=$ 0 . The polynomial $K$ is called the cofactor of the invariant algebraic hypersurface $f=0$. We remark that if the polynomial system has degree $m$, then any cofactor has at most degree $m-1$.

If $f=0$ and $g=0$ are two invariant algebraic hypersurfaces by the polynomial vector field $\mathcal{X}$, then $S=\{f=0\} \cap\{g=0\}$ is an invariant algebraic surface by the vector field $\mathcal{X}$, i.e. if an orbit of $\mathcal{X}$ has a point on the algebraic surface $S$, then the whole orbit is contained in $S$.

In the next theorem we characterize all the polynomial differential systems having the Clifford torus $\mathbb{T}$ as an invariant algebraic surface. 
Theorem 1. If the polynomial differential system (11) has the Clifford torus $\mathbb{T}$ invariant, then

$$
\begin{aligned}
& P_{1}=A\left(x_{1}^{2}+x_{2}^{2}-1 / 2\right)-2 C x_{2}, \\
& P_{2}=B\left(x_{1}^{2}+x_{2}^{2}-1 / 2\right)+2 C x_{1}, \\
& P_{3}=D\left(x_{3}^{2}+x_{4}^{2}-1 / 2\right)-2 F x_{4}, \\
& P_{4}=E\left(x_{3}^{2}+x_{4}^{2}-1 / 2\right)+2 F x_{3},
\end{aligned}
$$

where $A, B, C, D, E$ and $F$ are arbitrary polynomials in the variables $x_{1}, x_{2}, x_{3}$ and $x_{4}$.

Theorem 1 is proved in section 2 ,

For all $(a, b) \in \mathbb{R}^{2}$ such that $a^{2}+b^{2}=1 / 2$ a meridian of the Clifford torus $\mathbb{T}$ is

$$
M_{(a, b)}=\left\{\left(x_{1}, x_{2}, a, b\right) \in \mathbb{R}^{4}: x_{1}^{2}+x_{2}^{2}=1 / 2\right\},
$$

and a parallel is

$$
P_{(a, b)}=\left\{\left(a, b, x_{3}, x_{4}\right) \in \mathbb{R}^{4}: x_{3}^{2}+x_{4}^{2}=1 / 2\right\} .
$$

So a meridian is defined by the three equations $x_{1}^{2}+x_{2}^{2}=1 / 2, x_{3}=a$, $x_{4}=b$ with $a^{2}+b^{2}=1 / 2$, and similarly by a parallel.

In the next theorem we provide the maximum number of invariant meridians or parallels that a polynomial vector field $\mathcal{X}$ on $\mathbb{T}$ can have in function of its degree. See section 3 for the definition of the multiplicity of a meridian and of a parallel.

Theorem 2. Let $\mathcal{X}$ be a polynomial vector field on the Clifford torus $\mathbb{T}$ of degree $\mathbf{m}=\left(m_{1}, m_{2}, m_{3}, m_{4}\right)$ with $m_{1} \geqslant m_{2} \geqslant m_{3} \geqslant m_{4}>0$.

(a) The number of invariant meridians of $\mathcal{X}$ is at most $m_{4}-2$ taking into account their multiplicities if $m_{4}>3$, and 4 if $m_{4}=3$. These upper bounds are reached.

(b) The number of invariant parallels of $\mathcal{X}$ is at most $m_{2}-2$ taking into account their multiplicities if $m_{2}>3$, and 4 if $m_{2}=3$. These upper bounds are reached.

Theorem 2 is proved in section 3 ,

\section{Proof of Theorem 1}

For proving Theorem 1 we shall need some definitions and results for the polynomial differential systems in $\mathbb{R}^{2}$. 
Consider the following polynomial differential system in $\mathbb{R}^{2}$

$$
\begin{aligned}
& \dot{x}=P(x, y), \\
& \dot{y}=Q(x, y),
\end{aligned}
$$

and let

$$
\mathcal{Y}=P(x, y) \frac{\partial}{\partial x}+Q(x, y) \frac{\partial}{\partial y},
$$

its associated polynomial vector field.

Let $f(x, y)$ a polynomial. Then the algebraic curve $f(x, y)=0$ is invariant by system (5) if there exists a polynomial $k=k(x, y)$ such that $\mathcal{Y} f=k f$.

The next result is proved in Lemma 6 of [4].

Lemma 3. Assume that the polynomial system (15) has an invariant algebraic curve $f(x, y)=0$ without singularities (i.e. there are no points at which $f$ and its first derivatives are all vanish). If $\left(f_{x}, f_{y}\right)=1$ (i.e. the polynomials $f_{x}$ and $f_{y}$ has no common factors), then

$$
\begin{aligned}
& P=A f-C f_{y}, \\
& Q=B f+D f_{x},
\end{aligned}
$$

where $A, B$ and $C$ are arbitrary polynomials in the variables $x$ and $y$.

Proof of Theorem 1. We consider polynomial vector fields $\mathcal{X}$ given in (2) of degree $m$ in $\mathbb{R}^{4}$ having the Clifford torus $\mathbb{T}$ as an invariant algebraic surface, i.e. both hypersurfaces $x_{1}^{2}+x_{2}^{2}=1 / 2$ and $x_{3}^{2}+x_{4}^{2}=1 / 2$ are invariant by $\mathcal{X}$.

Let $f=x_{1}^{2}+x_{2}^{2}-1 / 2=0$ and $g=x_{3}^{2}+x_{4}^{2}-1 / 2=0$. By Lemma 3 and from the definition of the invariant algebraic hypersurface $f=0$ given in (3) it follows that

$$
\begin{aligned}
& P_{1}=A\left(x_{1}^{2}+x_{2}^{2}-1 / 2\right)-2 C x_{2}, \\
& P_{2}=B\left(x_{1}^{2}+x_{2}^{2}-1 / 2\right)+2 C x_{1},
\end{aligned}
$$

where $A, B$ and $C$ arbitrary polynomials in the variables $x_{1}, x_{2}, x_{3}$ and $x_{4}$. In a similar way, from Lemma 3 and the definition of the invariant algebraic hypersurface $g=0$ we get

$$
\begin{aligned}
& P_{3}=D\left(x_{3}^{2}+x_{4}^{2}-1 / 2\right)-2 F x_{4}, \\
& P_{4}=E\left(x_{3}^{2}+x_{4}^{2}-1 / 2\right)+2 F x_{3},
\end{aligned}
$$

where $D, E$ and $F$ arbitrary polynomials in the variables $x_{1}, x_{2}, x_{3}$ and $x_{4}$. In short from (6) and (7) the theorem follows. 


\section{Proof of Theorem 2}

One of the best tools for working with invariant algebraic hypersurfaces is the extactic polynomial of $\mathcal{X}$ associated to a finite vector space of polynomials generated by $W$. To our knowledge the extactic polynomial was introduced by Lagutinskii, see [11]. We recall its definition for a polynomial vector field in $\mathbb{R}^{4}$. Let $W$ be a finitely generated vector subspace of the vector space $\mathbb{C}\left[x_{1}, x_{2}, x_{3}, x_{d}\right]$ generated by the basis $\left\{v_{1}\right.$, $\left.\ldots, v_{l}\right\}$. The extactic polynomial of $\mathcal{X}$ associated to $W$ is

$\mathcal{E}_{W}(\mathcal{X})=\mathcal{E}_{\left\{v_{1}, \ldots, v_{l}\right\}}(\mathcal{X})=\operatorname{det}\left(\begin{array}{cccc}v_{1} & v_{2} & \ldots & v_{l} \\ \mathcal{X}\left(v_{1}\right) & \mathcal{X}\left(v_{2}\right) & \ldots & \mathcal{X}\left(v_{l}\right) \\ \vdots & \vdots & \ldots & \vdots \\ \mathcal{X}^{l-1}\left(v_{1}\right) & \mathcal{X}^{l-1}\left(v_{2}\right) & \ldots & \mathcal{X}^{l-1}\left(v_{l}\right)\end{array}\right)$

where $\mathcal{X}^{j}\left(v_{i}\right)=\mathcal{X}^{j-1}\left(\mathcal{X}\left(v_{i}\right)\right)$. The extactic polynomial does not dependent of the chosen basis of $W$.

The extactic polynomial $\mathcal{E}_{W}(\mathcal{X})$ has two good properties. First, it allows to detect invarian algebraic hypersurfaces $f=0$ with $f \in W$ by the polynomial vector field $\mathcal{X}$, see the following proposition proved in [3]. Second, it allows to compute the multiplicity of the invariant algebraic hypersurfaces.

Even if the next proposition is stated for complex polynomial vector fields, it is very useful for our later considerations. This is so, because we deal with real polynomial vector fields, which are particular cases of complex ones.

Proposition 4. Let $\mathcal{X}$ be a polynomial vector field in $\mathbb{C}^{4}$ and let $W$ be a finitely generated vector subspace of $\mathbb{C}\left[x_{1}, x_{2}, x_{3}, x_{4}\right]$ with $\operatorname{dim}(W)>1$. Then every algebraic invariant hypersurface $f=0$ for the vector field $\mathcal{X}$, with $f \in W$, is a factor of the polynomial $\mathcal{E}_{W}(\mathcal{X})$.

From Proposition 4 it follows that $f=0$ is an invariant hyperplane of the polynomial vector field $\mathcal{X}$ if the polynomial $f$ is a factor of the polynomial $\mathcal{E}_{W}(\mathcal{X})$, where $W$ is generated by $\left\{1, x_{1}, x_{2}, x_{3}, x_{4}\right\}$.

From [3] the invariant hypersurface $f=0$, with $f \in W$, has multiplicity $k$ if $k$ is the greatest positive integer such that $f^{k}$ divides the polynomial $\mathcal{E}_{W}(\mathcal{X})$. In [3] it is proved that if we have that $f=0$ is an invariante hypersurface of multiplicity $k$, then in a neighborhood of $\mathcal{X}$ in the topology of the coefficients there are polynomial vector fields $\mathcal{Y}_{\varepsilon}$, being $\varepsilon$ a small parameter, having $k$ invariant algebraic hypersurfaces such that all of them tend to the hypersurface $f=0$ when $\varepsilon \rightarrow 0$. 
We say that the meridian $M_{\left(a_{i}, b_{i}\right)}$ with $a_{i}^{2}+b_{i}^{2}=1 / 2$ has multiplicity $k$ if both invariant hyperplanes $x_{3}-a_{i}=0$ and $x_{4}-b_{i}=0$ of the differential system (1) with the polynomials $P_{i}$ given by (4) have multiplicities $k_{1}$ and $k_{2}$ respectively, and $\min \left\{k_{1}, k_{2}\right\}=k$. In a similar way is defined the multiplicity of a parallel $P_{\left(a_{i}, b_{i}\right)}$.

Proof of Theorem 2. A meridian of the Clifford torus $\mathbb{T}$ is obtained by intersecting $\mathbb{T}$ with the hyperplanes $x_{3}=a$ and $x_{4}=b$ with $a^{2}+b^{2}=$ $1 / 2$. So the hyperplanes $x_{3}-a=0$ and $x_{4}-b=0$ must be invariant by the polynomial vector field $\mathcal{X}$. In other words $x_{3}-a$ must divide the extactic polynomial

$$
\mathcal{E}_{1, x_{3}}(\mathcal{X})=\left|\begin{array}{ll}
1 & x_{3} \\
0 & P_{3}
\end{array}\right|=P_{3}
$$

i.e. $x_{3}-a$ must divide the polynomial $P_{3}\left(x_{1}, x_{2}, x_{3}, x_{4}\right)$. In a similar way $x_{4}-b$ must divide the polynomial $P_{4}\left(x_{1}, x_{2}, x_{3}, x_{4}\right)$. Since the degrees of $P_{i}$ is $m_{i}$ for $i=3$, 4 , it follows that the polynomials $x_{3}-a_{i}$ at most divide $m_{3}$ times the polynomial $P_{3}$. This is only possible if

$$
P_{3}=k_{3} \prod_{i=1}^{m_{3}}\left(x_{3}-a_{i}\right), \quad \text { and } \quad P_{4}=k_{4} \prod_{i=1}^{m_{4}}\left(x_{4}-b_{i}\right),
$$

with $k_{3}, k_{4} \in \mathbb{R} \backslash\{0\}$. But taking into account the expressions of $P_{3}$ and $P_{4}$ given in (4) we only can obtain the expressions of (8) if and only if

$$
D=2 k x_{3}, \quad E=-2 k x_{4}, \quad \text { and } \quad F=k x_{3} x_{4},
$$

with $k \in \mathbb{R} \backslash\{0\}$. Then

$$
\begin{aligned}
& P_{3}=2 k x_{3}\left(x_{3}^{2}+x_{4}^{2}-1 / 2\right)-2 k x_{3} x_{4}^{2}=2 k x_{3}\left(x_{3}^{2}-1 / 2\right), \\
& P_{4}=-2 k x_{4}\left(x_{3}^{2}+x_{4}^{2}-1 / 2\right)+2 k x_{3}^{2} x_{4}=2 k x_{4}\left(x_{4}^{2}-1 / 2\right) .
\end{aligned}
$$

So in this case $m_{3}=m_{4}=3$ and we have 4 meridians, namely $M_{(0,1 / \sqrt{2})}$, $M_{(0,-1 / \sqrt{2})}, M_{(1 / \sqrt{2}, 0)}$ and $M_{(-1 / \sqrt{2}, 0)}$.

Expect this exceptional case (9) that we can obtain for the polynomials $P_{3}$ and $P_{4}$ the expressions (8), we have that there are at most $m_{3}-2$ invariant hyperplanes of the form $x_{3}-b_{i}$ taking into account their multiplicities, and at most we have $m_{4}-2$ invariant hyperplanes of the form $x_{4}-b_{i}$ taking into account their multiplicities. These invariants hyperplanes are obtained when

$$
D=k_{3} \prod_{i=1}^{m_{3}-2}\left(x_{3}-a_{i}\right), \quad E=k_{4} \prod_{i^{1}}^{m_{4}-2}\left(x_{4}-b_{i}\right), \quad F=0
$$


with $k_{3}, k_{4} \in \mathbb{R} \backslash\{0\}$. Therefore the differential system (1) with the polynomials $P_{i}$ given by (41) has the invariant meridians $M_{\left(a_{i}, b_{i}\right)}$ if $a_{i}^{2}+$ $b_{i}^{2}=1 / 2$ for $i=1, \ldots, m_{4}-2$. Eventually some of these invariant meridians can have multiplicity larger than one if the $a_{i}$ and $b_{i}$ appears repeated in the expressions of the polynomials $D$ and $E$.

In short, if $m_{4}>3$ an upper bound for the maximum number of invariant meridians is $m_{4}-2$ taking into account their multiplicities, because $m_{3} \geq m_{4}$; or if $m_{4}=3$ that upper bound is 4 . Note from this proof that these upper bounds are reached. This proves statement $(a)$ of Theorem 2 .

In an analogous way we obtain that the maximum number of invariant parallels is $m_{2}-2$ taking into account their multiplicities if $m_{2}>3$, or 4 if $m_{2}=3$. This proves statement $(b)$ of Theorem 2.

\section{ACKNOWLEDGEMENTS}

The first author is partially supported by a FEDER-MINECO grant MTM2016-77278-P, a MINECO grant MTM2013-40998-P, and an AGAUR grant 2014SGR-568. The second author acknowledges support from a grant of the Romanian National Authority for Scientific Research and Innovation, CNCS-UEFISCDI, project number PN-II-RU-TE-2014-40657.

\section{REFERENCES}

[1] J.C. Artés, B. Grünbaum And J. Llibre, On the number of invariant straight lines for polynomial differential systems, Pacific J. of Math. 184 (1998), 207-230.

[2] A.A. De Barros, A.C. Brasil And L.A.M. De Sousa, A new characterization of submanifolds with parallel mean curvature vector in $S^{n+p}$, Kodai Math. J. 27 (2004), 45-56.

[3] C. Christopher, J. Llibre And J.V. Pereira, Multiplicity of invariant algebraic curves in polynomial vector fields, Pacific J. of Math. 229 (2007), 63-117.

[4] C. Christopher, J. Llibre, C. Pantazi and X. Zhang, Darboux integrability and invariant algebraic curves for planar polynomial systems, J. of Physics A: Math. and Gen. 35 (2002), 2457-2476.

[5] F.S. Dias, J. Llibre And L.F. Mello, When parallels and meridians are limit cycles for polynomial vector fields on quadrics of revolution in $\mathbb{R}^{3}$, Int. J. of Bifurcation and Chaos 26 (2016), 1650160-pp 14.

[6] U. Dursun and N.C. Turgay, Minimal and pseudo-umbilical rotational surfaces in Euclidean space $E^{4}$, Mediterr. J. Math. 10 (2013), 497-506.

[7] H. LI AND X. WANG, New characterizations of the Clifford torus as a Lagrangian self-shrinker, J. Geom. Anal. 27 (2017), 1393-1412. 
[8] J. Llibre And J.C. Medrado, On the invariant hyperplanes for $d$ dimensional polynomial vector fields, J. Phys. A: Math. Gen. 40 (2007), 83858391.

[9] J. Llibre AND J.C. Medrado, Limit cycles, invariant meridians and parallels for polynomial vector fields on the torus, Bulletin des Sciences Mathématiques 135 (2011), 1-9.

[10] J. Llibre AND S. REBOllo, Invariant parallels, invariant meridians and limit cycles of polynomial vector fields on some 2 -dimensional algebraic tori in $\mathbb{R}^{3}$, J. of Dynamics and Differential Equations 25 (2013), 777-793.

[11] J.M. Strelcyn And S. Wojciechowski, A method of finding integrals for three-dimensional dynamical systems, Phys. Lett. A 133 (1988), 207-212.

[12] J. ZHUANG AND Y. ZHANG, Submanifolds with parallel normalized mean curvature vector in a unit sphere, Arch. Math. (Basel) 96 (2011), no. 3, 281-290.

Jaume Llibre, Departament de Matemàtiques, Universitat Autònoma de Barcelona, 08193 Bellaterra, Barcelona, Catalonia, Spain

E-mail address: jllibre@mat.uab.cat

Adrian C. Murza, Institute of Mathematics "Simion Stoilow" of the Romanian Academy, Calea Griviţei 21, 010702 Bucharest, Romania

E-mail address: adrian_murza@hotmail.com 